\title{
The Existence and Uniqueness Result for a Relativistic Nonlinear Schrödinger Equation
}

\author{
Yongkuan Cheng and Jun Yang \\ Department of Mathematics, South China University of Technology, Guangzhou, Guangdong 510640, China \\ Correspondence should be addressed to Yongkuan Cheng; chengyk@scut.edu.cn \\ Received 3 August 2013; Revised 15 December 2013; Accepted 17 December 2013; Published 16 January 2014 \\ Academic Editor: Bernhard Ruf
}

Copyright (C) 2014 Y. Cheng and J. Yang. This is an open access article distributed under the Creative Commons Attribution License, which permits unrestricted use, distribution, and reproduction in any medium, provided the original work is properly cited.

We study the existence and uniqueness of positive solutions for a class of quasilinear elliptic equations. This model has been proposed in the self-channeling of a high-power ultrashort laser in matter.

\section{Introduction}

In this paper, we consider the following quasilinear Schrödinger equation:

$$
\begin{aligned}
&-\Delta u+\omega u-\frac{\kappa}{2}\left[\Delta\left(1+u^{2}\right)^{1 / 2}\right] \frac{u}{\left(1+u^{2}\right)^{1 / 2}} \\
&=|u|^{p-1} u, \quad x \in \mathbb{R}^{N},
\end{aligned}
$$

where $\omega>0, \kappa>0, N \geq 3$, and $2<p+1<2^{*}:=2 N /(N-2)$. Solutions of (1) are related to standing waves for the following quasilinear Schrödinger equation:

$$
\begin{array}{r}
i z_{t}=-\Delta z+W(x) z-h\left(|z|^{2}\right) z-\kappa \Delta l\left(|z|^{2}\right) l^{\prime}\left(|z|^{2}\right) z, \\
x \in \mathbb{R}^{N},
\end{array}
$$

where $z: \mathbb{R} \times \mathbb{R}^{N} \rightarrow \mathbb{C}, W: \mathbb{R}^{N} \rightarrow \mathbb{R}$ is a given potential, $\kappa$ is real constant, and $l$ and $h$ are real functions. Quasilinear equations such as (2) have been accepted as models of several physical phenomena corresponding to various types of $l$; see [1-5] for physical backgrounds.

The superfluid film equation in plasma physics has this structure for $l(s)=s$ (see [6]). Putting $z(t, x)=$ $\exp (-i E t) u(x)$, where $E \in \mathbb{R}$ and $u>0$ is a real function, (2) turns into the following equation:

$$
-\Delta u+V(x) u-\Delta\left(|u|^{2}\right) u=\rho(u), \quad x \in \mathbb{R}^{N},
$$

where $V(x)=W(x)-E$ is the new potential function and $\rho$ is the new nonlinearity. In this case, the first existence results are due to [7]. In [7], the main existence results are obtained through a constrained minimization argument. Subsequently, a general existence result was derived in [8]. The idea in [8] is to make a change of variables and reduce the quasilinear problem to semilinear one and Orlicz space framework was used to prove the existence of positive solutions via the Mountain pass theorem. The same method of changing of variables was also used in [9] but the usual Sobolev space $H^{1}\left(\mathbb{R}^{N}\right)$ framework was used as the working space. Precisely, since the energy functional associated (3) is not well defined in $H^{1}\left(\mathbb{R}^{N}\right)$, they first make the changing of unknown variables $v=f^{-1}(u)$, where $f$ is defined by ODE as follows:

$$
f^{\prime}(t)=\frac{1}{\sqrt{1+2 f^{2}(t)}}, \quad t \in[0,+\infty),
$$

and $f(t)=-f(-t), t \in(-\infty, 0]$. Then, after the changing of variable, to find the solutions of (2), it suffices to study the existence of solutions for the following semilinear equation:

$$
-\Delta v=\phi(x, v), \quad x \in \mathbb{R}^{N},
$$

where

$$
\phi(x, v)=\frac{1}{\sqrt{1+2 f^{2}(v)}}(-V(x) f(v)+\rho(f(v))) .
$$


By using the classical results given by [10], they proved the existence of a spherically symmetric solution. In [11], the authors give a sufficient condition for uniqueness of the ground state solutions by using the same change of variables as [9].

In the case $l(s)=(1+s)^{1 / 2},(2)$ models the self-channeling of a high-power ultrashort laser in matter (see [12]). In this case, few results are known. In [13], the authors proved global existence and uniqueness of small solutions in transverse space dimensions 2 and 3 and local existence without any smallness condition in transverse space dimension 1 . But they did not study the existence of standing waves. But we have to point out that the method of change of variables as (4) cannot be generalized to treat the case $l(s)=(1+s)^{1 / 2}$. In [14], the authors made the changing of known variable (see also [15])

$$
v=G(u)=\int_{0}^{u} \sqrt{1+2\left(s l^{\prime}\left(s^{2}\right)\right)^{2}} d s
$$

and proved the existence of nontrivial solution with $N \geq 3$ and $\kappa=1$. In this paper, for $l(s)=(1+s)^{1 / 2}$ and $\kappa>0$, we will show the existence and uniqueness result for (1) by using a change of variables due to $[14,15]$. One main difficulty in dealing with this problem seems to be that of obtaining the boundedness of a (PS) sequence for the corresponding functional. We overcome this difficulty by using Jeanjean's result [16].

Our main result is the following.

Theorem 1. Assume that $N \geq 3$, $\omega>0, \kappa>0$, and $\max \{4 \sqrt{2 /(2+\kappa)}-1,2\}<p+1<2^{*}$. There exists $c_{0}=$ $c_{0}(p, \kappa)>0$ such that if $\omega^{1 /(p-1)} \geq c_{0}$, then the positive solution of (1) is unique.

In this paper, $\mathrm{C}$ denotes positive (possibly different) constant, $L^{p}\left(\mathbb{R}^{N}\right)$ denotes the usual Lebesgue space with norm $\|u\|_{p}=\left(\int_{\mathbb{R}^{N}}|u|^{p} d x\right)^{1 / p}, 1 \leq p<\infty$, and $H^{1}\left(\mathbb{R}^{N}\right)$ denotes the Sobolev space with norm $\|u\|=\left(\int_{\mathbb{R}^{N}}\left(|\nabla u|^{2}+\right.\right.$ $\left.\left.\omega u^{2}\right) d x\right)^{1 / 2}$.

\section{Preliminaries}

We note that the solutions of (1) are the critical points of the following functional:

$$
\begin{aligned}
I(u)= & \frac{1}{2} \int_{\mathbb{R}^{N}}\left[1+\frac{\kappa u^{2}}{2\left(1+u^{2}\right)}\right]|\nabla u|^{2} d x \\
& +\frac{1}{2} \int_{\mathbb{R}^{N}} \omega u^{2} d x-\frac{1}{p+1} \int_{\mathbb{R}^{N}}|u|^{p+1} d x .
\end{aligned}
$$

Since the functional $I(u)$ may not be well defined in the usual Sobolev spaces $H^{1}\left(\mathbb{R}^{N}\right)$, we make a change of variables as

$$
v=G(u)=\int_{0}^{u} g(t) d t
$$

where $g(t)=\sqrt{1+\left(\kappa t^{2} / 2\left(1+t^{2}\right)\right)}$. Since $g(t)$ is monotonous with $|t|$, the inverse function $G^{-1}(t)$ of $G(t)$ exists. Then after the change of variables, $I(u)$ can be written as

$$
\begin{aligned}
J(v)= & \frac{1}{2} \int_{\mathbb{R}^{N}}|\nabla v|^{2} d x+\frac{1}{2} \int_{\mathbb{R}^{N}} \omega\left|G^{-1}(v)\right|^{2} d x \\
& -\frac{1}{p+1} \int_{\mathbb{R}^{N}}\left|G^{-1}(v)\right|^{p+1} d x .
\end{aligned}
$$

By Lemma 2 listed below, we have $\lim _{t \rightarrow 0} G^{-1}(t) / t=1$ and $\lim _{t \rightarrow \infty}\left|G^{-1}(t)\right| / t=\sqrt{2 /(2+\kappa)}$, so $J(v)$ is well defined in $H^{1}\left(\mathbb{R}^{N}\right)$ and $J(v) \in C^{1}$.

If $u$ is a nontrivial solution of (1), then for all $\phi \in C_{0}^{\infty}\left(\mathbb{R}^{N}\right)$ it should satisfy

$$
\begin{gathered}
\int_{\mathbb{R}^{N}}\left[g^{2}(u) \nabla u \nabla \phi+g(u) g^{\prime}(u)|\nabla u|^{2} \phi\right. \\
\left.+\omega u \phi-u^{p} \phi\right] d x=0 .
\end{gathered}
$$

We show that (11) is equivalent to

$$
\begin{array}{r}
J^{\prime}(v) \psi \\
=\int_{\mathbb{R}^{N}}\left[\nabla v \nabla \psi+\omega \frac{G^{-1}(v)}{g\left(G^{-1}(v)\right)} \psi-\frac{\left|G^{-1}(v)\right|^{p}}{g\left(G^{-1}(v)\right)} \psi\right] d x=0, \\
\forall \psi \in C_{0}^{\infty}\left(\mathbb{R}^{N}\right) .
\end{array}
$$

Indeed, if we choose $\phi=(1 / g(u)) \psi$ in (11), then we get (12). On the other hand, since $u=G^{-1}(v)$, if we let $\psi=$ $g(u) \phi$ in (12), we get (11). Therefore, in order to find the nontrivial solutions of (1), it suffices to study the existence of the nontrivial solutions of the following equation:

$$
-\Delta v=\frac{\left|G^{-1}(v)\right|^{p}}{g\left(G^{-1}(v)\right)}-\omega \frac{G^{-1}(v)}{g\left(G^{-1}(v)\right)}, \quad x \in \mathbb{R}^{N} .
$$

Before we close this section, we give some properties of the change of variables.

Lemma 2. (1) $\sqrt{2 /(2+\kappa)} t \leq\left|G^{-1}(t)\right| \leq t$ for all $t \geq 0$;

(2) $\left|\left(G^{-1}(t)\right)^{\prime}\right| \leq 1$ for all $t \in \mathbb{R}$;

(3) $\lim _{t \rightarrow 0}\left(\left|G^{-1}(t)\right| / t\right)=1$;

(4) $\lim _{t \rightarrow \infty}\left(\left|G^{-1}(t)\right| / t\right)=\sqrt{2 /(2+\kappa)}$;

(5) $\operatorname{tg}^{\prime}(t) / g(t) \leq((\kappa+4)-2 \sqrt{2(2+\kappa)}) / \kappa$ for all $t \in \mathbb{R}$;

(6) $\sqrt{2 /(2+\kappa)} G^{-1}(t) \leq t\left(G^{-1}(t)\right)^{\prime} \leq G^{-1}(t)$ for all $t \geq 0$.

Proof. (1) Since $\left[G^{-1}(t)-(1 / g(0)) t\right]^{\prime}=1 / g\left(G^{-1}(t)\right)-$ $1 / g(0) \leq 0$ and $\left[G^{-1}(t)-(1 / g(\infty)) t\right]^{\prime}=1 / g\left(G^{-1}(t)\right)-$ $1 / g(\infty) \geq 0$, so $1 / g(\infty) t \leq G^{-1}(t) \leq(1 / g(0)) t$, for $t \geq 0$; that is, $(1 / g(\infty)) t=\sqrt{2 /(2+\kappa)} t \leq G^{-1}(t) \leq(1 / g(0)) t=t$, for $t \geq 0$, which proves (1).

Since $\lim _{t \rightarrow 0}\left(G^{-1}(t) / t\right)=\left(\left.\left(G^{-1}(t)\right)^{\prime}\right|_{t=0}=1 / g\left(G^{-1}(0)\right)=\right.$ 1 and $g(t)$ is increasing, so properties (2) and (3) are obvious. 
For (4), the result is obvious since $g(t)$ is an increasing bounded function.

Since

$$
\begin{aligned}
\frac{t}{g(t)} g^{\prime}(t) & =\frac{\kappa t^{2}}{2\left(1+t^{2}\right)^{2} g^{2}(t)} \\
& =\frac{\kappa t^{2}}{2+(\kappa+4) t^{2}+(\kappa+2) t^{4}} \\
& =\frac{\kappa}{2 / t^{2}+(\kappa+4)+(\kappa+2) t^{2}} \\
& \leq \frac{(\kappa+4)-2 \sqrt{2(2+\kappa)}}{\kappa}
\end{aligned}
$$

which proves (5).

For (6), since $g$ is a increasing function, then $G(t) \leq$ $g(t) t$, which implies that $t\left(G^{-1}(t)\right)^{\prime} \leq G^{-1}(t)$. On the other hand, by (1) and $\sqrt{2 /(2+\kappa)} \leq\left(G^{-1}(t)\right)^{\prime} \leq 1$, we get $\sqrt{2 /(2+\kappa)} G^{-1}(t) \leq t\left(G^{-1}(t)\right)^{\prime}$.

\section{Existence}

At first, we give two Lemmas.

Lemma 3. There exist $\rho_{0}, a_{0}>0$ such that $J(v) \geq a_{0}$ for all $\|v\|=\rho_{0}$.

Proof. Let

$$
Q(t):=-\frac{1}{2} \omega\left|G^{-1}(t)\right|^{2}+\frac{1}{p+1}\left|G^{-1}(t)\right|^{p+1} .
$$

Then, by Lemma 2 and $2<p+1<2^{*}$, we have

$$
\begin{aligned}
\lim _{t \rightarrow 0} \frac{Q(t)}{t^{2}}=\lim _{t \rightarrow 0}[ & -\frac{1}{2} \omega\left(\frac{G^{-1}(t)}{t}\right)^{2} \\
& \left.+\frac{1}{p+1}\left(\frac{G^{-1}(t)}{t}\right)^{2}\left|G^{-1}(t)\right|^{p-1}\right] \\
= & -\frac{1}{2} \omega, \\
\lim _{t \rightarrow \infty} \frac{Q(t)}{t^{2^{*}}=} \lim _{t \rightarrow \infty}\left[-\frac{1}{2} \omega\left(\frac{\left|G^{-1}(t)\right|}{t}\right)^{2} \frac{1}{t^{2^{*}-2}}\right. & \left.+\frac{1}{p+1}\left(\frac{\left|G^{-1}(t)\right|}{t}\right)^{p+1} \frac{1}{t^{2^{*}-(p+1)}}\right]=0 .
\end{aligned}
$$

Thus, for $\epsilon>0$ sufficiently small, there exists a constant $C_{\epsilon}>$ 0 such that

$$
Q(t) \leq\left(-\frac{1}{2} \omega+\epsilon\right) t^{2}+C_{\epsilon}|t|^{2^{*}}
$$

Then, we have

$$
\begin{aligned}
J(v)= & \frac{1}{2} \int_{\mathbb{R}^{N}}|\nabla v|^{2} d x+\frac{1}{2} \int_{\mathbb{R}^{N}} \omega\left|G^{-1}(v)\right|^{2} d x \\
& -\frac{1}{p+1} \int_{\mathbb{R}^{N}}\left|G^{-1}(v)\right|^{p+1} d x \\
\geq & \frac{1}{2} \int_{\mathbb{R}^{N}}|\nabla v|^{2} d x+\frac{1}{2} \int_{\mathbb{R}^{N}} \omega v^{2} d x-\epsilon \\
& \times \int_{\mathbb{R}^{N}} v^{2} d x-C_{\epsilon} \int_{\mathbb{R}^{N}} v^{2^{*}} d x \\
\geq & C\|v\|^{2}-C\|v\|^{2^{*}} .
\end{aligned}
$$

Thus, by choosing $\rho_{0}$ small, we get the result when $\|v\|=$ $\rho_{0}$.

Lemma 4. There exists $v \in H^{1}\left(\mathbb{R}^{N}\right)$ such that $J(v)<0$.

Proof. Given $\phi \in C_{0}^{\infty}\left(\mathbb{R}^{N},[0,1]\right)$ with supp $\phi:=\bar{B}_{1}$, we will prove that $J(s \phi) \rightarrow-\infty$ as $s \rightarrow \infty$, which will prove the result if we take $v=s \phi$ with $s$ large enough. By Lemma 2, we have $G^{-1}(t) \geq C t$ as $t \geq 1$, so

$$
\begin{aligned}
J(s \phi) \leq & \frac{1}{2} s^{2} \int_{\mathbb{R}^{N}}|\nabla \phi|^{2} d x+\frac{1}{2} s^{2} \int_{\mathbb{R}^{N}} \omega \phi^{2} d x-s^{p+1} C \\
& \times \int_{\{|s \phi| \geq 1\}} \phi^{p+1} d x \longrightarrow-\infty,
\end{aligned}
$$

as $s \rightarrow \infty$. Thus, we get the result.

We will use the following Theorem which is due to Jeanjean [16].

Theorem 5. Let $X$ be a Banach space equipped with the norm $\|\cdot\|$ and let $L \subset \mathbb{R}^{+}$be an interval. One considers a family $\left(I_{\lambda}\right)_{\lambda \in L}$ of $C^{1}$-functionals on $X$ of the form

$$
I_{\lambda}(u)=A(u)-\lambda B(u), \quad \forall \lambda \in L,
$$

where $B(u) \geq 0$, for all $u \in X$, and such that either $A(u) \rightarrow$ $+\infty$ or $B(u) \rightarrow+\infty$ as $\|u\| \rightarrow \infty$. One assumes that there are two points $\left(v_{1}, v_{2}\right)$ in $X$ such that setting

$$
\Gamma=\left\{\gamma \in C([0,1], X), \gamma(0)=v_{1}, \gamma(1)=v_{2}\right\},
$$

there hold, for all $\lambda \in L$,

$$
c_{\lambda}:=\inf _{\gamma \in \Gamma \in[0,1]} \max _{\lambda}(\gamma(t))>\max \left\{I_{\lambda}\left(v_{1}\right), I_{\lambda}\left(v_{2}\right)\right\} .
$$

Then, for almost every $\lambda \in L$, there is a subsequence $\left\{v_{n}(\lambda)\right\} \subset$ $X$ such that

(i) $\left\{v_{n}(\lambda)\right\}$ is bounded;

(ii) $I_{\lambda}\left(v_{n}(\lambda)\right) \rightarrow c_{\lambda}$;

(iii) $I_{\lambda}^{\prime}\left(v_{n}(\lambda)\right) \rightarrow 0$ in the dual $X^{-1}$ of $X$. 
We consider the functional

$$
\begin{aligned}
J_{\lambda}(v)= & \frac{1}{2} \int_{\mathbb{R}^{N}}\left(|\nabla v|^{2}+\omega\left|G^{-1}(v)\right|^{2}\right) d x \\
& -\frac{\lambda}{p+1} \int_{\mathbb{R}^{N}}\left|G^{-1}(v)\right|^{p+1} d x,
\end{aligned}
$$

where $\lambda \in[1 / 2,1]$.

Let $L=[1 / 2,1]$. We find that

$$
B(v):=\frac{1}{p+1} \int_{\mathbb{R}^{N}}\left|G^{-1}(v)\right|^{p+1} d x \geq 0
$$

for all $v \in H^{1}\left(\mathbb{R}^{N}\right)$. On the other hand, if $\|v\|=\left(\int_{\mathbb{R}^{N}}\left(|\nabla v|^{2}+\right.\right.$ $\left.\left.\omega v^{2}\right) d x\right)^{1 / 2} \rightarrow+\infty$, then either $\int_{\mathbb{R}^{N}}|\nabla v|^{2} d x \rightarrow+\infty$, which implies $A(v):=\int_{\mathbb{R}^{N}}\left(|\nabla v|^{2}+\omega\left|G^{-1}(v)\right|^{2}\right) d x \rightarrow+\infty$, or $\int_{\mathbb{R}^{N}} \omega v^{2} d x \rightarrow+\infty$; in this case, to verify that $A(v) \rightarrow+\infty$, we start splitting

$$
\int_{\mathbb{R}^{N}} \omega v^{2} d x=\int_{\{x:|v(x)| \geq 1\}} \omega v^{2} d x+\int_{\{x:|v(x)| \leq 1\}} \omega v^{2} d x,
$$

since

$$
\begin{gathered}
\int_{\{x:|v(x)| \geq 1\}} \omega v^{2} d x \leq C \int_{\{x:|v(x)| \geq 1\}} v^{2^{*}} d x \\
\leq C\left(\int_{\mathbb{R}^{N}}|\nabla v|^{2} d x\right)^{2^{*} / 2},
\end{gathered}
$$

and by Lemma $2(6)$, we have $v=G\left(G^{-1}(v)\right) \leq g\left(G^{-1}(v)\right)$ $G^{-1}(v)$, so

$$
\int_{\{x:|v(x)| \leq 1\}} \omega\left|G^{-1}(v)\right|^{2} d x \geq \frac{1}{g^{2}(1)} \int_{\{x:|v(x)| \leq 1\}} \omega v^{2} d x,
$$

so $A(v) \rightarrow+\infty$.

For $J_{\lambda}(v)$ defined above with $\lambda \in[1 / 2,1]$, using Lemma 4, we get a $v \in H^{1}\left(\mathbb{R}^{N}\right)$ such that $J_{\lambda}(v)<0$. Also from Lemma 2 we know that $B(v)=o\left(\|v\|^{2}\right)$ as $v \rightarrow 0$. Thus setting

$$
\Gamma=\left\{\gamma \in C\left([0,1], H^{1}\left(\mathbb{R}^{N}\right)\right), \gamma(0)=0, \gamma(1)=v\right\},
$$

we have, for all $\lambda \in[1 / 2,1]$,

$$
c_{\lambda}:=\inf _{\gamma \in \Gamma \in[0,1]} \max _{\lambda} J_{\lambda}(\gamma(t))>0=\max \left\{J_{\lambda}(0), J_{\lambda}(v)\right\} .
$$

Therefore, using Theorem 5 , for almost all $\lambda \in[1 / 2,1]$, there exists a subsequence $\left\{v_{n}(\lambda)\right\} \subset H^{1}\left(\mathbb{R}^{N}\right)$ such that

(i) $\left\{v_{n}(\lambda)\right\}$ is bounded in $H^{1}\left(\mathbb{R}^{N}\right)$;

(ii) $J_{\lambda}\left(v_{n}(\lambda)\right) \rightarrow c_{\lambda}$;

(iii) $J_{\lambda}^{\prime}\left(v_{n}(\lambda)\right) \rightarrow 0$ in $H^{-1}\left(\mathbb{R}^{N}\right)$.

Lemma 6. Assume that $\left\{v_{n}(\lambda)\right\} \subset H^{1}\left(\mathbb{R}^{N}\right)$ is a bounded Palais-Smale sequence of the functional $J_{\lambda}$ for $\lambda \in[1 / 2,1]$. Then there exists a nontrivial critical point of $J_{\lambda}$.
Proof. We first note that $\left\{v_{n}(\lambda)\right\} \subset H^{1}\left(\mathbb{R}^{N}\right)$ satisfies

$$
\begin{aligned}
J_{\lambda}\left(v_{n}(\lambda)\right)= & \frac{1}{2} \int_{\mathbb{R}^{N}}\left|\nabla v_{n}(\lambda)\right|^{2} d x \\
& +\frac{1}{2} \int_{\mathbb{R}^{N}} \omega\left|G^{-1}\left(v_{n}(\lambda)\right)\right|^{2} d x \\
& -\frac{\lambda}{p+1} \int_{\mathbb{R}^{N}}\left|G^{-1}\left(v_{n}(\lambda)\right)\right|^{p+1} d x=c_{\lambda}+o(1),
\end{aligned}
$$

and, for any $\psi \in C_{0}^{\infty}\left(\mathbb{R}^{N}\right)$,

$$
\begin{aligned}
J_{\lambda}^{\prime}\left(v_{n}(\lambda)\right) \psi=\int_{\mathbb{R}^{N}} & {\left[\nabla v_{n}(\lambda) \nabla \psi+\omega \frac{G^{-1}\left(v_{n}(\lambda)\right)}{g\left(G^{-1}\left(v_{n}(\lambda)\right)\right)} \psi\right.} \\
& \left.-\lambda \frac{\left|G^{-1}\left(v_{n}(\lambda)\right)\right|^{p}}{g\left(G^{-1}\left(v_{n}(\lambda)\right)\right)} \psi\right] d x=o(1)\|\psi\| .
\end{aligned}
$$

Since $\left\{v_{n}(\lambda)\right\}$ is a bounded Palais-Smale sequence, there exists $v(\lambda) \in H^{1}\left(\mathbb{R}^{N}\right)$ such that $v_{n}(\lambda) \rightarrow v(\lambda)$ in $H^{1}\left(\mathbb{R}^{N}\right)$ and $v_{n}(\lambda) \rightarrow v(\lambda)$ in $L_{\text {loc }}^{p}\left(\mathbb{R}^{N}\right)$ for $p \in\left[2,2^{*}\right)$. By the Lebesgue Dominated Theorem, we have

$$
\begin{aligned}
& J_{\lambda}^{\prime}\left(v_{n}(\lambda)\right) \psi-J_{\lambda}^{\prime}(v(\lambda)) \psi \\
& =\int_{\mathbb{R}^{N}}\left(\nabla v_{n}(\lambda)-\nabla v(\lambda)\right) \nabla \psi d x \\
& \quad+\omega \int_{\mathbb{R}^{N}}\left[\frac{G^{-1}\left(v_{n}(\lambda)\right)}{g\left(G^{-1}\left(v_{n}(\lambda)\right)\right)}-\frac{G^{-1}(v)}{g\left(G^{-1}(v(\lambda))\right)}\right] \psi d x \\
& \quad-\lambda \int_{\mathbb{R}^{N}}\left[\frac{\left|G^{-1}\left(v_{n}(\lambda)\right)\right|^{p}}{g\left(G^{-1}\left(v_{n}(\lambda)\right)\right)}-\frac{\left|G^{-1}(v(\lambda))\right|^{p}}{g\left(G^{-1}(v(\lambda))\right)}\right] \psi d x \\
& \quad \longrightarrow 0 .
\end{aligned}
$$

Hence, $v$ is a weak solution of $(1)$. If $v(\lambda) \not \equiv 0$, then we get the result.

Otherwise, if $v(\lambda)=0$, we claim that for all $R>0$,

$$
\lim _{n \rightarrow \infty} \sup _{y \in \mathbb{R}^{N}} \int_{B_{R}(y)} v_{n}(\lambda)^{2} d x=0
$$

cannot occur. Suppose by contradiction that (33) occurs, that is, $\left\{v_{n}(\lambda)\right\}$ vanish; then, by the Lions compactness Lemma (see $[17,18]), v_{n} \rightarrow 0$ in $L^{r}\left(\mathbb{R}^{N}\right)$ for any $r \in\left(2,2^{*}\right)$. Since $2<$ $p+1<2^{*}$, then by the proof of Lemma 2 , we get

$$
\left|\frac{\left|G^{-1}(t)\right|^{p}}{g\left(G^{-1}(t)\right)} t\right| \leq\left|G^{-1}(t)\right|^{p+1} \leq \varepsilon t^{2}+C_{\varepsilon}|t|^{p+1}
$$


which implies that

$$
\begin{gathered}
0=\lim _{n \rightarrow \infty} J_{\lambda}^{\prime}\left(v_{n}(\lambda)\right) v_{n}(\lambda) \\
=\lim _{n \rightarrow \infty} \int_{\mathbb{R}^{N}}\left[\left|\nabla v_{n}(\lambda)\right|^{2}+\omega \frac{G^{-1}\left(v_{n}(\lambda)\right)}{g\left(G^{-1}\left(v_{n}(\lambda)\right)\right)} v_{n}(\lambda)\right. \\
\left.-\lambda \frac{\left|G^{-1}\left(v_{n}(\lambda)\right)\right|^{p}}{g\left(G^{-1}\left(v_{n}(\lambda)\right)\right)} v_{n}(\lambda)\right] d x \\
\geq \lim _{n \rightarrow \infty} \int_{\mathbb{R}^{N}}\left[\left|\nabla v_{n}(\lambda)\right|^{2}+\omega \frac{G^{-1}\left(v_{n}(\lambda)\right)}{g\left(G^{-1}\left(v_{n}(\lambda)\right)\right)} v_{n}(\lambda)\right. \\
\left.-\lambda \varepsilon v_{n}(\lambda)^{2}-\lambda C_{\varepsilon}\left|v_{n}(\lambda)\right|^{p+1}\right] d x .
\end{gathered}
$$

Since $\varepsilon \rightarrow 0$ and $p+1 \in\left(2,2^{*}\right)$, then

$$
\begin{gathered}
\lim _{n \rightarrow \infty} \int_{\mathbb{R}^{N}}\left|\nabla v_{n}(\lambda)\right|^{2} d x=0, \\
\lim _{n \rightarrow \infty} \int_{\mathbb{R}^{N}} \omega \frac{G^{-1}\left(v_{n}(\lambda)\right)}{g\left(G^{-1}\left(v_{n}(\lambda)\right)\right)} v_{n}(\lambda) d x=0 .
\end{gathered}
$$

On the other hand, note by Lemma 2 (5) that

$$
\begin{aligned}
\left|\nabla\left(G^{-1}\left(v_{n}(\lambda)\right)\right) g\left(G^{-1}\left(v_{n}(\lambda)\right)\right)\right| \\
\quad \leq\left[1+\frac{G^{-1}\left(v_{n}(\lambda)\right)}{g\left(G^{-1}\left(v_{n}(\lambda)\right)\right)} g^{\prime}\left(G^{-1}\left(v_{n}(\lambda)\right)\right)\right]\left|\nabla v_{n}(\lambda)\right| \\
\quad \leq \frac{2(\kappa+2)-2 \sqrt{2(2+\kappa)}}{\kappa}\left|\nabla v_{n}(\lambda)\right| .
\end{aligned}
$$

Combing Lemma 2, we have $G^{-1}\left(v_{n}(\lambda)\right) g\left(G^{-1}\left(v_{n}(\lambda)\right)\right) \in$ $H^{1}\left(\mathbb{R}^{N}\right)$. In fact, we only need to show that $\mid G^{-1}\left(v_{n}(\lambda)\right) g\left(G^{-1}\left(v_{n}(\lambda)\right)|\leq| v_{n}(\lambda) \mid\right.$; let $\Phi(t)=g(t) t-G(t)$; then by Lemma 2 (5), we have

$$
\begin{array}{r}
\Phi^{\prime}(t)=g^{\prime}(t) t-2 g(t) \leq \frac{4-\kappa-2 \sqrt{2(2+\kappa)}}{\kappa} g(t)<0, \\
\text { for } \kappa>0,
\end{array}
$$

so $g(t) t \leq G(t)$ for $t \geq 0$ and $g(t) t \geq G(t)$ for $t<0$, which implies that $\left|G^{-1}\left(v_{n}(\lambda)\right) g\left(G^{-1}\left(v_{n}(\lambda)\right)\right)\right| \leq\left|v_{n}(\lambda)\right|$. Thus, since $C_{0}^{\infty}\left(\mathbb{R}^{N}\right)$ is dense in $H^{1}\left(\mathbb{R}^{N}\right)$, by choosing

$$
\psi=G^{-1}\left(v_{n}(\lambda)\right) g\left(G^{-1}\left(v_{n}(\lambda)\right)\right)
$$

in (31), we deduce that

$$
\begin{aligned}
o(1)\left\|v_{n}(\lambda)\right\| \\
=J_{\lambda}^{\prime}\left(v_{n}(\lambda)\right) G^{-1}\left(v_{n}(\lambda)\right) g\left(G^{-1}\left(v_{n}(\lambda)\right)\right) \\
=\int_{\mathbb{R}^{N}}\left[\left(1+\frac{G^{-1}\left(v_{n}(\lambda)\right)}{g\left(G^{-1}\left(v_{n}(\lambda)\right)\right)} g^{\prime}\left(G^{-1}\left(v_{n}(\lambda)\right)\right)\right)\right. \\
\times\left|\nabla v_{n}(\lambda)\right|^{2} \\
\left.+\omega\left|G^{-1}\left(v_{n}(\lambda)\right)\right|^{2}-\lambda\left|G^{-1}\left(v_{n}(\lambda)\right)\right|^{p+1}\right] d x .
\end{aligned}
$$

So

$$
\lim _{n \rightarrow \infty} \int_{\mathbb{R}^{N}} \omega\left|G^{-1}\left(v_{n}(\lambda)\right)\right|^{2} d x=0
$$

Combing (36) and (34), we have

$$
\begin{aligned}
& J_{\lambda}\left(v_{n}(\lambda)\right)= \frac{1}{2} \int_{\mathbb{R}^{N}}\left|\nabla v_{n}(\lambda)\right|^{2} d x \\
&+\frac{1}{2} \int_{\mathbb{R}^{N}} \omega\left|G^{-1}\left(v_{n}(\lambda)\right)\right|^{2} d x \\
&-\frac{\lambda}{p+1} \int_{\mathbb{R}^{N}}\left|G^{-1}\left(v_{n}(\lambda)\right)\right|^{p+1} d x \longrightarrow 0, \\
& \text { as } n \longrightarrow \infty,
\end{aligned}
$$

so we get a contradiction since $J_{\lambda}\left(v_{n}(\lambda)\right) \rightarrow c_{\lambda}>0$. Thus, $\left\{v_{n}(\lambda)\right\}$ does not vanish and there exist $k, R>0$, and $\left\{y_{n}\right\} \subset$ $\mathbb{R}^{N}$ such that

$$
\lim _{n \rightarrow \infty} \int_{B_{R}\left(y_{n}\right)} v_{n}(\lambda)^{2} d x \geq k>0 .
$$

Define $v_{n}(\lambda):=v_{n}^{\lambda}$ and $\widetilde{v}_{n}^{\lambda}(x)=v_{n}^{\lambda}\left(x+y_{n}\right)$. Since $\left\{v_{n}^{\lambda}\right\}$ is a Palais-Smale sequence for $J_{\lambda},\left\{\widetilde{v}_{n}^{\lambda}\right\}$ is also a Palais-Smale sequence for $J_{\lambda}$ with $J_{\lambda}^{\prime}\left(\widetilde{v}^{\lambda}\right)=0$ if $\widetilde{v}_{n}^{\lambda} \rightarrow \widetilde{v}^{\lambda}$ in $H^{1}\left(\mathbb{R}^{N}\right)$. Since $\left\{\widetilde{v}_{n}^{\lambda}\right\}$ does not vanish, we have that $\widetilde{v}^{\lambda} \neq 0$ is a nontrivial solution of (1).

From Lemma 6, we see that, for almost all $\lambda \in[1 / 2,1]$, there exists a solution $v(\lambda)$ to the following Schrödinger equation:

$$
-\Delta v=\tilde{h}(x, v), \quad x \in \mathbb{R}^{N},
$$

where

$$
\tilde{h}(x, v)=-\omega \frac{G^{-1}(v)}{g\left(G^{-1}(v)\right)}+\lambda \frac{\left|G^{-1}(v)\right|^{p}}{g\left(G^{-1}(v)\right)} .
$$

Therefore, we can choose $\left\{\lambda_{j}\right\} \subset[1 / 2,1]$ such that $\lambda_{j} \rightarrow$ 1. Setting $v_{j}:=v\left(\lambda_{j}\right)$, we have $J_{\lambda_{j}}^{\prime}\left(v_{j}\right)=0$. We can deduce that $v$ is a solution to (13) if we show that $J^{\prime}(v)=0$. To prove this, in view of Lemma 6 , we first check that $\left\{v_{j}\right\}$ is bounded in $H^{1}\left(\mathbb{R}^{N}\right)$. 
Notice that the Pohozaev identity implies that the solutions of (45) satisfy

$$
\begin{gathered}
\frac{N-2}{2 N} \int_{\mathbb{R}^{N}}|\nabla v|^{2} d x+\frac{\omega}{2} \int_{\mathbb{R}^{N}}\left|G^{-1}(v)\right|^{2} d x \\
=\frac{\lambda}{p+1} \int_{\mathbb{R}^{N}}\left|G^{-1}(v)\right|^{p+1} d x .
\end{gathered}
$$

Lemma 7. The sequence $\left\{v_{j}\right\}$ is bounded.

Proof. Since $v_{j}$ is a solution to (45) with $\lambda=\lambda_{j}$, by (47), we have

$$
\begin{aligned}
c_{1 / 2} \geq & c_{\lambda_{j}} \\
= & \frac{1}{2} \int_{\mathbb{R}^{N}}\left|\nabla v_{j}\right|^{2} d x+\frac{1}{2} \int_{\mathbb{R}^{N}} \omega\left|G^{-1}\left(v_{j}\right)\right|^{2} d x \\
& -\frac{\lambda_{j}}{p+1} \int_{\mathbb{R}^{N}}\left|G^{-1}\left(v_{j}\right)\right|^{p+1} d x \\
= & \frac{1}{2} \int_{\mathbb{R}^{N}}\left|\nabla v_{j}\right|^{2} d x+\frac{1}{2} \int_{\mathbb{R}^{N}} \omega\left|G^{-1}\left(v_{j}\right)\right|^{2} d x \\
& -\left[\frac{N-2}{2 N} \int_{\mathbb{R}^{N}}\left|\nabla v_{j}\right|^{2} d x+\frac{\omega}{2} \int_{\mathbb{R}^{N}}\left|G^{-1}\left(v_{j}\right)\right|^{2} d x\right] \\
\geq & \frac{1}{N} \int_{\mathbb{R}^{N}}\left|\nabla v_{j}\right|^{2} d x,
\end{aligned}
$$

which implies that $\int_{\mathbb{R}^{N}}\left|\nabla v_{j}\right|^{2} d x$ is bounded. On the other hand, together with (41), we have

$$
\begin{aligned}
(p+1) c_{\lambda_{j}}-0= & (p+1) J_{\lambda_{j}}\left(v_{j}\right) \\
& -J_{\lambda_{j}}^{\prime}\left(v_{j}\right) G^{-1}\left(v_{j}\right) g\left(G^{-1}\left(v_{j}\right)\right) \\
\geq & \left(\frac{p+1}{2}-\frac{2(\kappa+2)-2 \sqrt{2(2+\kappa)}}{\kappa}\right) \\
& \times \int_{\mathbb{R}^{N}}\left|\nabla v_{j}\right|^{2} d x \\
& +\frac{p-1}{2} \int_{\mathbb{R}^{N}} \omega\left|G^{-1}\left(v_{j}\right)\right|^{2} d x .
\end{aligned}
$$

Since $\int_{\mathbb{R}^{N}}\left|\nabla v_{j}\right|^{2} d x$ is bounded, so $\int_{\mathbb{R}^{N}} \omega\left|G^{-1}\left(v_{j}\right)\right|^{2} d x$ is bounded. To verify that $\left\{v_{j}\right\}$ is bounded in $H^{1}\left(\mathbb{R}^{N}\right)$, we start splitting

$$
\int_{\mathbb{R}^{N}} \omega v_{j}^{2} d x=\int_{\left\{x:\left|v_{j}\right| \geq 1\right\}} \omega v_{j}^{2} d x+\int_{\left\{x:\left|v_{j}\right| \leq 1\right\}} \omega v_{j}^{2} d x,
$$

since

$$
\begin{gathered}
\int_{\left\{x:\left|v_{j}\right| \geq 1\right\}} \omega v_{j}^{2} d x \leq C \int_{\left\{x:\left|v_{j}\right| \geq 1\right\}} v_{j}^{2^{*}} d x \leq C\left(\int_{\mathbb{R}^{N}}\left|\nabla v_{j}\right|^{2} d x\right)^{2^{*} / 2}, \\
\int_{\left\{x:\left|v_{j}\right| \leq 1\right\}} \omega\left|G^{-1}\left(v_{j}\right)\right|^{2} d x \geq \frac{1}{g^{2}(1)} \int_{\left\{x:\left|v_{j}\right| \leq 1\right\}} \omega v_{j}^{2} d x,
\end{gathered}
$$

so $\left\|v_{j}\right\|=\left(\int_{\mathbb{R}^{N}}\left(\left|\nabla v_{j}\right|^{2}+\omega v_{j}^{2}\right) d x\right)^{1 / 2}$ is bounded.
Lemma 8. Assume that $N \geq 3, \omega>0$, and $2<p+1<2^{*}$. Then (1) has a nontrivial solution.

Proof. The boundedness of $\left\{v_{j}\right\}$ in $H^{1}\left(\mathbb{R}^{N}\right)$ follows from Lemma 7; we have that $\left\{G^{-1}\left(v_{j}\right)\right\}$ is bounded in $L^{s}\left(\mathbb{R}^{N}\right)$ for $2 \leq s \leq 2^{*}$. Then for any $\psi \in C_{0}^{\infty}\left(\mathbb{R}^{N}\right)$, we have

$$
\begin{aligned}
J_{\lambda_{j}}^{\prime}\left(v_{j}\right) \psi-J^{\prime}\left(v_{j}\right) \psi \\
=\left(1-\lambda_{j}\right) \int_{\mathbb{R}^{N}} \frac{\left|G^{-1}\left(v_{j}\right)\right|^{p}}{g\left(G^{-1}\left(v_{j}\right)\right)} \psi d x \\
\leq\left(1-\lambda_{j}\right) \int_{\mathbb{R}^{N}}\left|G^{-1}\left(v_{j}\right)\right|^{p} \psi d x \\
\leq\left(1-\lambda_{j}\right)\left(\int_{\mathbb{R}^{N}}\left|G^{-1}\left(v_{j}\right)\right|^{p \times((p+1) / p)}\right)^{p /(p+1)} \\
\quad \times\left(\int_{\mathbb{R}^{N}} \psi^{p+1}\right)^{1 /(p+1)},
\end{aligned}
$$

since

$$
\left(\int_{\mathbb{R}^{N}}\left|G^{-1}\left(v_{j}\right)\right|^{p \times((p+1) / p)}\right)^{p /(p+1)}\left(\int_{\mathbb{R}^{N}} \psi^{p+1}\right)^{1 /(p+1)}<\infty,
$$

so $J_{\lambda_{j}}^{\prime}\left(v_{j}\right) \psi-J^{\prime}\left(v_{j}\right) \psi \rightarrow 0$ as $j \rightarrow \infty$; thus we have $J^{\prime}\left(v_{j}\right) \rightarrow$ 0 as $j \rightarrow \infty$. By knowing that

$$
\begin{aligned}
J\left(v_{j}\right)= & J_{\lambda_{j}}\left(v_{j}\right)-\frac{\left(1-\lambda_{j}\right)}{p+1} \\
& \times \int_{\mathbb{R}^{N}}\left|G^{-1}\left(v_{j}\right)\right|^{p+1} d x,
\end{aligned}
$$

since

$$
\frac{\left(1-\lambda_{j}\right)}{p+1} \iint_{\mathbb{R}^{N}}\left|G^{-1}\left(v_{j}\right)\right|^{p+1} d x \longrightarrow 0,
$$

so $\limsup _{j \rightarrow \infty} J\left(v_{j}\right)=\limsup _{j \rightarrow \infty} J_{\lambda_{j}}\left(v_{j}\right)$, and we distinguish two cases. Either $\limsup _{j \rightarrow \infty} J_{\lambda_{j}}\left(v_{j}\right)>0$ or $\limsup _{j \rightarrow \infty} J_{\lambda_{j}}\left(v_{j}\right) \leq 0$. In the first case, we get $\limsup _{j \rightarrow \infty} J\left(v_{j}\right):=c>0$ and the result follows from Lemma 6.

In the second case, we define the sequence $\left\{z_{j}\right\} \subset H^{1}\left(\mathbb{R}^{N}\right)$ by $z_{j}=t_{j} v_{j}$ with $t_{j} \in[0,1]$ satisfying

$$
J_{\lambda_{j}}\left(z_{j}\right)=\max _{t \in[0,1]} J_{\lambda_{j}}\left(t v_{j}\right)
$$


(if for a $j \in \mathbb{N}, t_{j}$ defined by (56) is not unique, we choose the smaller possible value). By construction $\left\{z_{j}\right\} \subset H^{1}\left(\mathbb{R}^{N}\right)$ is bounded. Moreover by the definition of (56), we have

$$
\begin{gathered}
0=\left.\frac{d J_{\lambda_{j}}\left(t v_{j}\right)}{d t}\right|_{t=t_{j}} \\
=\frac{1}{t_{j}}\left[\int_{\mathbb{R}^{N}}\left|\nabla z_{j}\right|^{2} d x+\int_{\mathbb{R}^{N}} \omega \frac{G^{-1}\left(z_{j}\right)}{g\left(G^{-1}\left(z_{j}\right)\right)} z_{j} d x\right. \\
\left.\quad-\lambda_{j} \frac{\left|G^{-1}\left(z_{j}\right)\right|^{p}}{g\left(G^{-1}\left(z_{j}\right)\right)} z_{j} d x\right],
\end{gathered}
$$

so $J_{\lambda_{j}}^{\prime}\left(z_{j}\right) z_{j}=0$. Then following the proof above, we have $J^{\prime}\left(z_{j}\right) \rightarrow 0$ and $\liminf \inf _{j \rightarrow \infty} J\left(z_{j}\right)=\liminf _{j \rightarrow \infty} J_{\lambda_{j}}\left(z_{j}\right)$. On the other hand, by the proof of Lemmas 3 and 2 (6), there exists a constant $C>0$ such that $J_{\lambda_{j}}^{\prime}(v) v \geq C\|v\|^{2}+o\left(\|v\|^{2}\right)$ as $v \rightarrow 0$, uniformly in $j \in \mathbb{N}$. Thus, since $J_{\lambda_{j}}^{\prime}\left(v_{j}\right)=0$, there is $\alpha>0$ such that $\left\|v_{j}\right\| \geq \alpha$, for all $j \in \mathbb{N}$. Similarly, following the proof of Lemma 3, we have $J_{\lambda_{j}}(v) \geq C\|v\|^{2}+o\left(\|v\|^{2}\right)$ with $C>0$ as $v \rightarrow 0$. Then recording that $\limsup _{j \rightarrow \infty} J_{\lambda_{j}}\left(v_{j}\right) \leq 0$, we obtain from (56) that

$$
\liminf _{j \rightarrow \infty} J\left(z_{j}\right)=\liminf _{j \rightarrow \infty} J_{\lambda_{j}}\left(z_{j}\right):=c^{\prime}>0 .
$$

Using Lemma 6 again, we complete the proof of Lemma 8 which implies that $u=G^{-1}(v)$ is a solution for (1).

Remark 9. In [14], the authors considered the existence of solutions for the following quasilinear Schrödinger equation:

$$
\begin{array}{r}
-\operatorname{div}\left(g^{2}(u) \nabla u\right)+g(u) g^{\prime}(u)|\nabla u|^{2}+V(x) u=h(u), \\
x \in \mathbb{R}^{N},
\end{array}
$$

where the nonlinearity $h$ is Hölder continuous and satisfies the following conditions:

$$
\begin{aligned}
& \left(h_{0}\right) h(s)=0 \text { if } s \leq 0 ; \\
& \left(h_{1}\right) h(s)=o(s) \text { as } s \rightarrow 0^{+} ; \\
& \left(h_{2}\right) \text { there exists } 2<p<2^{*}:=2 N /(N-2) \text { such that } \\
& |h(s)| \leq C\left(1+g(s)|G(s)|^{p-1}\right) \text {; } \\
& \left(h_{3}\right) \text { there exists } \mu>2 \text { such that for any } s>0 \text {, there } \\
& \text { holds } 0<\mu g(s) H(s) \leq G(s) h(s) .
\end{aligned}
$$

If we take $g^{2}(t)=1+\kappa t^{2} / 2\left(1+t^{2}\right), V(x) \equiv \omega$, and $h(u)=$ $|u|^{p-1} u$, (59) turns into (1) with $\kappa=1$. We point out that the existence result in [14] does not cover our result.

Now, we show that $\left(h_{3}\right)$ is not satisfied for $h(u)=|u|^{p-1} u$ if $2<p+1<2^{*}$. In fact, $0<\mu g(s) H(s) \leq G(s) h(s)$ if and only if

$$
\frac{\mu H(s)}{s h(s)} \leq \frac{G(s)}{s g(s)}
$$

By Lemma 2 (5), we have

$$
\frac{G(s)}{s g(s)} \geq \frac{\kappa}{2 \kappa+4-2 \sqrt{2(2+\kappa)}} .
$$

Thus, we only need to show

$$
\frac{\mu H(s)}{s h(s)} \leq \frac{\kappa}{2 \kappa+4-2 \sqrt{2(2+\kappa)}}
$$

that is

$$
\frac{2 \kappa+4-2 \sqrt{2(2+\kappa)}}{\kappa} \mu \leq p+1,
$$

under the hypothesis $h(s)=s^{p}$. Then, by (63), we have

$$
\begin{gathered}
2<2(6-2 \sqrt{6})<p+1<2^{*}, \quad \kappa=1, \\
4<p+1<2^{*}, \quad \kappa \longrightarrow \infty .
\end{gathered}
$$

Remark 10. In [14], $\left(h_{3}\right)$ is used to prove the boundedness of (PS) sequence. In this paper, since $\left(h_{3}\right)$ does not satisfy our condition, we obtain the boundedness of (PS) sequence by using Jeanjean's result [16].

\section{Uniqueness}

In this section, we study the uniqueness of the positive radial solution of (13). We put

$$
\begin{gathered}
f(s):=\frac{\left|G^{-1}(s)\right|^{p}}{g\left(G^{-1}(s)\right)}-\omega \frac{G^{-1}(s)}{g\left(G^{-1}(s)\right)}, \\
\text { for } s \geq 0, \quad K_{f}(s):=\frac{s f^{\prime}(s)}{f(s)} .
\end{gathered}
$$

We apply the following uniqueness result due to Serrin and Tang [19].

Theorem 11. Suppose that there exists $b>0$ such that

(1) $f$ is continuous on $(0, \infty), f(s) \leq 0$ on $(0, b]$, and $f(s)>0$ for $s>b$;

(2) $f \in C^{1}(b, \infty)$ and $K_{f}^{\prime}(s) \leq 0$ on $(b, \infty)$.

Then the semilinear problem

$$
\begin{gathered}
-\Delta u=f(u) \text { in } \mathbb{R}^{N}, \quad u>0, \\
u \longrightarrow 0 \text { as }|x| \longrightarrow \infty, \quad u(0)=\max u(x),
\end{gathered}
$$

has at most one positive radial solution.

Now we can see that $f$ defined in (65) is of the class $C^{1}[0, \infty)$. Moreover, by the proof of Lemma 2, we have that $G^{-1}(s)$ is increasing and $G^{-1}(0)=0$; then $G^{-1}(s) \geq 0$. 
So $-\omega+\left|G^{-1}(s)\right|^{p-1} \geq-\omega$; then there exists a unique $b$ such that $-\omega+\left|G^{-1}(b)\right|^{p-1}=0$, and

$$
\begin{array}{r}
f(s)=\frac{G^{-1}(s)}{g\left(G^{-1}(s)\right)}\left(-\omega+\left|G^{-1}(s)\right|^{p-1}\right) \leq 0, \\
\text { for } s \in(0, b], \\
f(s)=\frac{G^{-1}(s)}{g\left(G^{-1}(s)\right)}\left(-\omega+\left|G^{-1}(s)\right|^{p-1}\right)>0, \\
\quad \text { for } s \in(b,+\infty) .
\end{array}
$$

So (1) of Theorem 11 holds. From $-\omega+\left|G^{-1}(b)\right|^{p-1}=0$, we can also observe that $b=G\left(\omega^{1 /(p-1)}\right)$. Since $G(s)$ is increasing and $\lim _{s \rightarrow \infty}(G(s) / s)=\sqrt{(2+\kappa) / 2}$, this implies that

$$
b \longrightarrow \infty \quad \text { iff } \omega^{1 /(p-1)} \longrightarrow \infty .
$$

Lemma 12. Suppose $N \geq 3$ and $p>4 \sqrt{2 /(2+\kappa)}-2$. Then there exists $c_{0}=c_{0}(p, \kappa)>0$ such that if $\omega^{1 /(p-1)} \geq c_{0}$, then $f$ satisfies (2) of Theorem 11.

Proof. We observe that

$$
K_{f}^{\prime}(s)=\frac{1}{f(s)^{2}}\left(s f(s) f^{\prime \prime}(s)+f(s) f^{\prime}(s)-s f^{\prime}(s)^{2}\right) .
$$

Thus we have only to show that $s f f^{\prime \prime}+f f^{\prime}-s f^{\prime 2}<0$, for $s>b$. Since

$$
f(s)=\frac{\left(G^{-1}(s)\right)^{p}-\omega G^{-1}(s)}{g\left(G^{-1}(s)\right)},
$$

so

$$
\begin{aligned}
f^{\prime}(s)= & \frac{1}{g^{2}\left(G^{-1}(s)\right)}\left[p\left(G^{-1}(s)\right)^{p-1}-\omega\right]-\frac{g^{\prime}\left(G^{-1}(s)\right)}{g^{3}\left(G^{-1}(s)\right)} \\
& \times\left[\left(G^{-1}(s)\right)^{p}-\omega G^{-1}(s)\right], \\
f^{\prime \prime}(s)= & \frac{1}{g^{3}\left(G^{-1}(s)\right)} p(p-1)\left(G^{-1}(s)\right)^{p-2} \\
& -\frac{3 g^{\prime}\left(G^{-1}(s)\right)}{g^{4}\left(G^{-1}(s)\right)}\left[p\left(G^{-1}(s)\right)^{p-1}-\omega\right] \\
& -\left[\frac{g^{\prime \prime}\left(G^{-1}(s)\right)}{g^{4}\left(G^{-1}(s)\right)}-\frac{3\left(g^{\prime}\left(G^{-1}(s)\right)\right)^{2}}{g^{5}\left(G^{-1}(s)\right)}\right] \\
& \times\left[\left(G^{-1}(s)\right)^{p}-\omega G^{-1}(s)\right] .
\end{aligned}
$$

Then by complicated computations, we have

$$
\begin{aligned}
& s f(s) f^{\prime \prime}(s)+f(s) f^{\prime}(s)-s\left(f^{\prime}(s)\right)^{2} \\
&= \frac{1}{g^{3}\left(G^{-1}(s)\right)}\left[\left(G^{-1}(s)\right)^{p}-\omega G^{-1}(s)\right] \\
& \times \frac{p(p-1) s\left(G^{-1}(s)\right)^{p-2}}{g\left(G^{-1}(s)\right)}+\left[p\left(G^{-1}(s)\right)^{p-1}-\omega\right] \\
&-\frac{s}{g\left(G^{-1}(s)\right)} \frac{\left[p\left(G^{-1}(s)\right)^{p-1}-\omega\right]^{2}}{(s))^{p}-\omega G^{-1}(s)} \\
&-\frac{s g^{\prime \prime}\left(G^{-1}(s)\right)}{g^{2}\left(G^{-1}(s)\right)}\left[\left(G^{-1}(s)\right)^{p}-\omega G^{-1}(s)\right] \\
&-\frac{s g^{\prime}\left(G^{-1}(s)\right)}{g^{2}\left(G^{-1}(s)\right)}\left[p\left(G^{-1}(s)\right)^{p-1}-\omega\right] \\
&-\frac{g^{\prime}\left(G^{-1}(s)\right)}{g\left(G^{-1}(s)\right)}\left[\left(G^{-1}(s)\right)^{p}-\omega G^{-1}(s)\right] \\
&\left.+\frac{2 s\left(g^{\prime}\left(G^{-1}(s)\right)\right)^{2}}{g^{3}\left(G^{-1}(s)\right)}\left[\left(G^{-1}(s)\right)^{p}-\omega G^{-1}(s)\right]\right) \\
&=\frac{1}{g^{3}\left(G^{-1}(s)\right)}\left[\left(G^{-1}(s)\right)^{p}-\omega G^{-1}(s)\right]\left(H_{1}(s)+H_{2}(s)\right),
\end{aligned}
$$

where

$$
\begin{aligned}
H_{1}(s):= & \frac{p(p-1) s\left(G^{-1}(s)\right)^{p-2}}{g\left(G^{-1}(s)\right)}+\left[p\left(G^{-1}(s)\right)^{p-1}-\omega\right] \\
& -\frac{s}{g\left(G^{-1}(s)\right)} \frac{\left[p\left(G^{-1}(s)\right)^{p-1}-\omega\right]^{2}}{\left(G^{-1}(s)\right)^{p}-\omega G^{-1}(s)}, \\
H_{2}(s):= & -\frac{s g^{\prime \prime}\left(G^{-1}(s)\right)}{g^{2}\left(G^{-1}(s)\right)}\left[\left(G^{-1}(s)\right)^{p}-\omega G^{-1}(s)\right] \\
& -\frac{s g^{\prime}\left(G^{-1}(s)\right)}{g^{2}\left(G^{-1}(s)\right)}\left[p\left(G^{-1}(s)\right)^{p-1}-\omega\right] \\
& -\frac{g^{\prime}\left(G^{-1}(s)\right)}{g\left(G^{-1}(s)\right)}\left[\left(G^{-1}(s)\right)^{p}-\omega G^{-1}(s)\right] \\
& +\frac{2 s\left(g^{\prime}\left(G^{-1}(s)\right)\right)^{2}}{g^{3}\left(G^{-1}(s)\right)}\left[\left(G^{-1}(s)\right)^{p}-\omega G^{-1}(s)\right] .
\end{aligned}
$$

For $s>b$, it follows that $\left(1 / g^{3}\left(G^{-1}(s)\right)\right)\left[\left(G^{-1}(s)\right)^{p}-\right.$ $\left.\omega G^{-1}(s)\right]>0$. Thus it suffices to show that $H_{1}(s)+H_{2}(s)<0$, for $s>b$, in order to prove that $s f(s) f^{\prime \prime}(s)+f(s) f^{\prime}(s)-$ $s\left(f^{\prime}(s)\right)^{2}<0$. 
By (4) of Lemma 2 and $\lim _{t \rightarrow \infty} g(t)=\sqrt{(2+\kappa) / 2}$, we have

$$
\begin{aligned}
\lim _{s \rightarrow \infty} \frac{H_{1}(s)}{s^{p-1}}= & \lim _{s \rightarrow \infty} \frac{p(p-1)}{g\left(G^{-1}(s)\right)} \frac{\left(G^{-1}(s)\right)^{p-2}}{s^{p-2}} \\
& +\lim _{s \rightarrow \infty} p \frac{\left(G^{-1}(s)\right)^{p-1}-\omega}{s^{p-1}} \\
& -\lim _{s \rightarrow \infty} \frac{1}{g\left(G^{-1}(s)\right)} \\
& \times \frac{\left[p\left(G^{-1}(s)\right)^{p-1}-\omega\right]^{2}}{s^{p-2}\left[\left(G^{-1}(s)\right)^{p}-\omega G^{-1}(s)\right]} \\
= & \left(\sqrt{\frac{2}{2+\kappa}}\right)^{p-1}\left(p(p-1)+p-p^{2}\right)=0 .
\end{aligned}
$$

Thus, for sufficiently large $b$, we obtain $H_{1}(s)+H_{2}(s)<0$ if and only if $\mathrm{H}_{2}(s)<0$ for $s>b$.

Next, we investigate the sign of $\mathrm{H}_{2}(s)$. Firstly, we express $g^{\prime \prime}$ in terms of $g$ and $g^{\prime}$, and since $g(t)=\sqrt{1+\kappa t^{2} / 2\left(1+t^{2}\right)}$, so $g^{\prime}(t)=(1 / 2 g(t))\left(\kappa t /\left(1+t^{2}\right)^{2}\right)$ and

$$
\begin{aligned}
g^{\prime \prime}(t)= & -\frac{g^{\prime}(t)}{2 g^{2}(t)} \frac{\kappa t}{\left(1+t^{2}\right)^{2}}+\frac{1}{2 g(t)} \frac{\kappa}{\left(1+t^{2}\right)^{2}} \\
& -\frac{2 t}{1+t^{2}} \frac{1}{g(t)} \frac{\kappa t}{\left(1+t^{2}\right)^{2}} \\
= & -\frac{\left(g^{\prime}(t)\right)^{2}}{g(t)}+\frac{g^{\prime}(t)}{t}-\frac{4 t g^{\prime}(t)}{1+t^{2}} .
\end{aligned}
$$

Thus we obtain

$$
\begin{aligned}
H_{2}(s)= & {\left[\frac{3 s\left(g^{\prime}\left(G^{-1}(s)\right)\right)^{2}}{g^{3}\left(G^{-1}(s)\right)}-\frac{s g^{\prime}\left(G^{-1}(s)\right)}{G^{-1}(s) g^{2}\left(G^{-1}(s)\right)}\right.} \\
& \left.+\frac{4 s G^{-1}(s) g^{\prime}\left(G^{-1}(s)\right)}{\left[1+\left(G^{-1}(s)\right)^{2}\right] g^{3}\left(G^{-1}(s)\right)}\right] \\
& \times\left[\left(G^{-1}(s)\right)^{p}-\omega G^{-1}(s)\right] \\
& -\frac{s g^{\prime}\left(G^{-1}(s)\right)}{g^{2}\left(G^{-1}(s)\right)}\left[p\left(G^{-1}(s)\right)^{p-1}-\omega\right] \\
& -\frac{g^{\prime}\left(G^{-1}(s)\right)}{g\left(G^{-1}(s)\right)}\left[\left(G^{-1}(s)\right)^{p}-\omega G^{-1}(s)\right] .
\end{aligned}
$$

We note that

$$
\begin{aligned}
& \lim _{s \rightarrow \infty} \frac{\left(G^{-1}(s)\right)^{p}-\omega G^{-1}(s)}{s^{p}}=\left(\sqrt{\frac{2}{2+\kappa}}\right)^{p}, \\
& \lim _{s \rightarrow \infty} \frac{p\left(G^{-1}(s)\right)^{p-1}-\omega}{s^{p-1}}=p\left(\sqrt{\frac{2}{2+\kappa}}\right)^{p-1},
\end{aligned}
$$

so

$$
\begin{aligned}
& \lim _{s \rightarrow \infty} \frac{H_{2}(s)}{s^{p-3}} \\
& =\left(\sqrt{\frac{2}{2+\kappa}}\right)^{p}\left[\lim _{s \rightarrow \infty} \frac{3 s^{4}\left(g^{\prime}\left(G^{-1}(s)\right)\right)^{2}}{g^{3}\left(G^{-1}(s)\right)}\right. \\
& \quad-\lim _{s \rightarrow \infty} \frac{s^{4} g^{\prime}\left(G^{-1}(s)\right)}{G^{-1}(s) g^{2}\left(G^{-1}(s)\right)} \\
& +\lim _{s \rightarrow \infty} \frac{4 s^{4} G^{-1}(s) g^{\prime}\left(G^{-1}(s)\right)}{\left[1+\left(G^{-1}(s)\right)^{2}\right] g^{3}\left(G^{-1}(s)\right)} \\
& \left.-\lim _{s \rightarrow \infty} \frac{s^{3} g^{\prime}\left(G^{-1}(s)\right)}{g\left(G^{-1}(s)\right)}\right] \\
& -p\left(\sqrt{\frac{2}{2+\kappa}}\right)^{p-1} \lim _{s \rightarrow \infty} \frac{s^{3} g^{\prime}\left(G^{-1}(s)\right)}{g^{2}\left(G^{-1}(s)\right)} .
\end{aligned}
$$

Moreover, we have $G^{-1}(s) \sim \sqrt{2 /(2+\kappa)} s, g\left(G^{-1}(s)\right) \sim$ $\sqrt{(2+\kappa) / 2}$, and $g^{\prime}\left(G^{-1}(s)\right) \sim(\kappa(2+\kappa) / 4) s^{-3}$ as $s \rightarrow \infty$. Then, from $p>4 \sqrt{2 /(2+\kappa)}-2$, we have

$$
\begin{aligned}
\lim _{s \rightarrow \infty} \frac{H_{2}(s)}{s^{p-3}}= & \left(\sqrt{\frac{2}{2+\kappa}}\right)^{p} \\
& \times\left[-\frac{\kappa}{2} \sqrt{\frac{2+\kappa}{2}}+2 \kappa-\frac{\kappa(\kappa+2)}{4} \sqrt{\frac{2}{2+\kappa}}\right] \\
& -p\left(\sqrt{\frac{2}{2+\kappa}}\right)^{p-1} \frac{\kappa}{2} \\
= & \left(\sqrt{\frac{2}{2+\kappa}}\right)^{p-1} \kappa\left[2 \sqrt{\frac{2}{2+\kappa}}-1-\frac{p}{2}\right]<0,
\end{aligned}
$$

so $H_{2}(s)<0$ for $s>b$ if $b$ is sufficiently large; that is, $H_{1}(s)+$ $H_{2}(s)<0$ for $s>b$. From (68), there exists $c_{0}=c_{0}(p, \kappa)>$ 0 such that if $\omega^{1 /(p-1)} \geq c_{0}$, then we obtain $s f(s) f^{\prime \prime}(s)+$ $f(s) f^{\prime}(s)-s\left(f^{\prime}(s)\right)^{2}<0$ for $s>b=G\left(\omega^{1 /(p-1)}\right)$.

By Lemma 8, we can apply Theorem 11, Hence we obtain the uniqueness of positive radial solutions of (13). 


\section{Conclusion}

By the discussion of Section 3, we have a nontrivial solution of (1). Then using the result of Gidas et al. [20], we know that the nontrivial solution is a positive radial solution with $u \rightarrow$ 0 as $|x| \rightarrow \infty$ and $u(0)=\max u(x)$. Combined with the discussion of Section 4 , we complete the proof of Theorem 1 . That is, if $N \geq 3, \kappa>0, \omega>0$, and $\max \{4 \sqrt{2 /(2+\kappa)}-1,2\}<$ $p+1<2^{*}$, there exists $c_{0}(p, \kappa)>0$ such that if $\omega^{1 /(p-1)} \geq$ $c_{0}(p, \kappa)$, then the positive solution of (1) is unique.

\section{Conflict of Interests}

The authors declare that there is no conflict of interests regarding the publication of this paper.

\section{Acknowledgment}

This paper is supported by NSFC (nos. 11201154 and 11371146) and the Fundamental Research Funds for the Central Universities (nos. 2013ZM0112 and 2013ZM0113).

\section{References}

[1] A. G. Litvak and A. M. Sergeev, "One dimensional collapse of plasma waves," JETP Letters, vol. 27, pp. 517-520, 1978.

[2] R. W. Hasse, "A general method for the solution of nonlinear soliton and kink Schrödinger equations," Zeitschrift für Physik $B$, vol. 37, no. 1 , pp. 83-87, 1980.

[3] A. M. Kosevich, B. A. Ivanov, and A. S. Kovalev, "Magnetic solitons," Physics Report, vol. 194, no. 3-4, pp. 117-238, 1990.

[4] G. R. W. Quispel and H. W. Capel, "Equation of motion for the Heisenberg spin chain," Physica A, vol. 110, no. 1-2, pp. 41-80, 1982.

[5] B. Hartmann and W. Zakzewski, "Electrons on hexagonal lattices and applications to nanotubes," Physical Review, vol. 68, pp. 1-9, 2003.

[6] S. Kurihara, "Large-amplitude quasi-solitons in superfluid films," Journal of the Physical Society of Japan, vol. 50, no. 10, pp. 3262-3267, 1981.

[7] M. Poppenberg, K. Schmitt, and Z. Q. Wang, "On the existence of soliton solutions to quasilinear Schrödinger equations," Calculus of Variations and Partial Differential Equations, vol. 14, no. 3, pp. 329-344, 2002.

[8] J. Q. Liu, Y. Q. Wang, and Z. Q. Wang, "Soliton solutions for quasilinear Schrödinger equations, II," Journal of Differential Equations, vol. 187, no. 2, pp. 473-493, 2003.

[9] M. Colin and L. Jeanjean, "Solutions for a quasilinear Schrödinger equation: a dual approach," Nonlinear Analysis, Theory, Methods \& Applications, vol. 56, no. 2, pp. 213-226, 2004.

[10] H. Berestycki and P.-L. Lions, "Nonlinear scalar field equations, I existence of a ground state," Archive for Rational Mechanics and Analysis, vol. 82, no. 4, pp. 313-345, 1983.

[11] S. Adachi and T. Watanabe, "Uniqueness of the ground state solutions of quasilinear Schrödinger equations," Nonlinear Analysis, Theory, Methods \& Applications, vol. 75, no. 2, pp. 819833, 2012.
[12] E. W. Laedke, K. H. Spatschek, and L. Stenflo, "Evolution theorem for a class of perturbed envelope soliton solutions," Journal of Mathematical Physics, vol. 24, no. 12, pp. 2764-2769, 1982.

[13] A. De Bouard, N. Hayashi, and J. Saut, "Global existence of small solutions to a relativistic nonlinear Schrödinger equation," Communications in Mathematical Physics, vol. 189, no. 1, pp. 73105, 1997.

[14] Y. Shen and Y. Wang, "Soliton solutions for generalized quasilinear Schrödinger equations," Nonlinear Analysis: Theory, Methods \& Applications, vol. 80, pp. 194-201, 2013.

[15] S. Yaotian and G. Xinkang, "The positive solution of degenerate variational problems and degenerate elliptic equations," Chinese Journal of Contemporary Mathematics, vol. 14, no. 2, 1993.

[16] L. Jeanjean, "On the existence of bounded Palais-Smale sequences and application to a Landesman-Lazer-type problem," Proceedings of the Royal Society of Edinburgh A, vol. 129, no. 4, pp. 787-809, 1999.

[17] P. L. Lions, "The concentration compactness principle in the calculus of variations. The locally compact case. Part I," AnnaLes de L'Institut Henri Poincaré, vol. 1, no. 1, pp. 109-145, 1984.

[18] P. L. Lions, "The concentration compactness principle in the calculus of variations. The locally compact case. Part II," AnnaLes de L'Institut Henri Poincaré, vol. 1, no. 4, pp. 223-283, 1984.

[19] J. Serrin and M. Tang, "Uniqueness of ground states for quasilinear elliptic equations," Indiana University Mathematics Journal, vol. 49, no. 3, pp. 897-923, 2000.

[20] B. Gidas, N. Wei-Ming, and L. Nirenberg, "Symmetry and related properties via the maximum principle," Communications in Mathematical Physics, vol. 68, no. 3, pp. 209-243, 1979. 


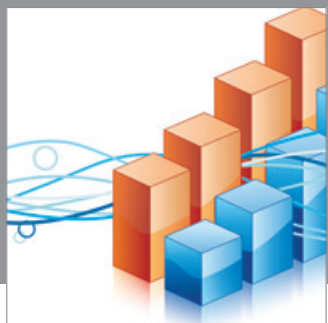

Advances in

Operations Research

mansans

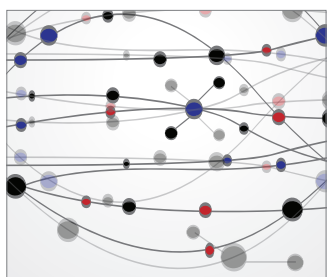

The Scientific World Journal
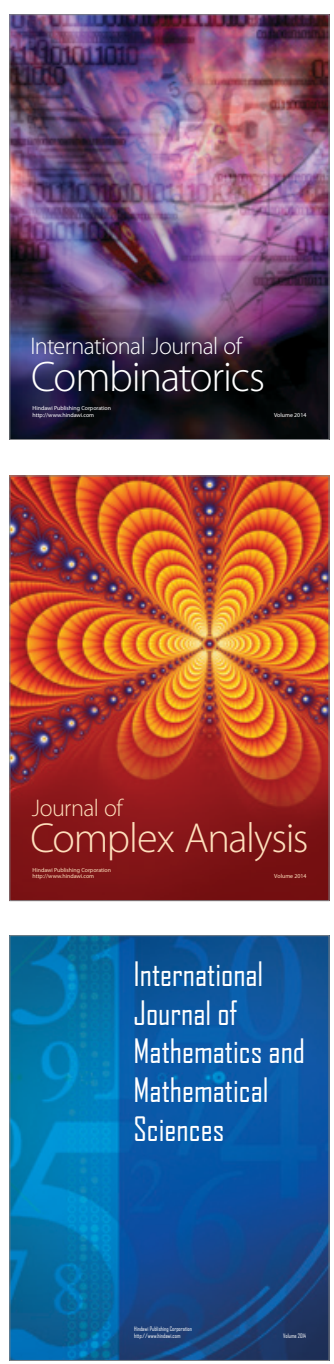
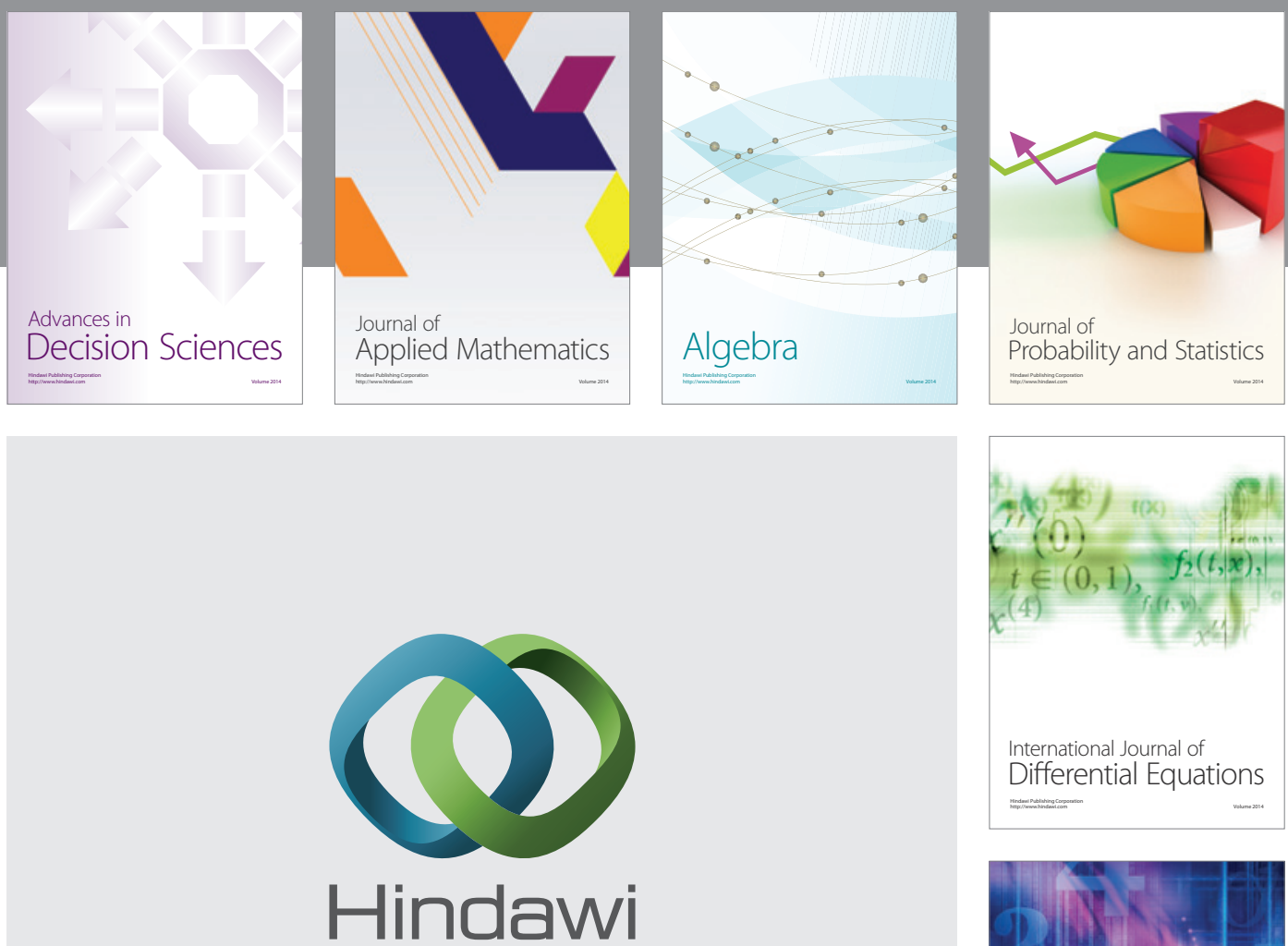

Submit your manuscripts at http://www.hindawi.com
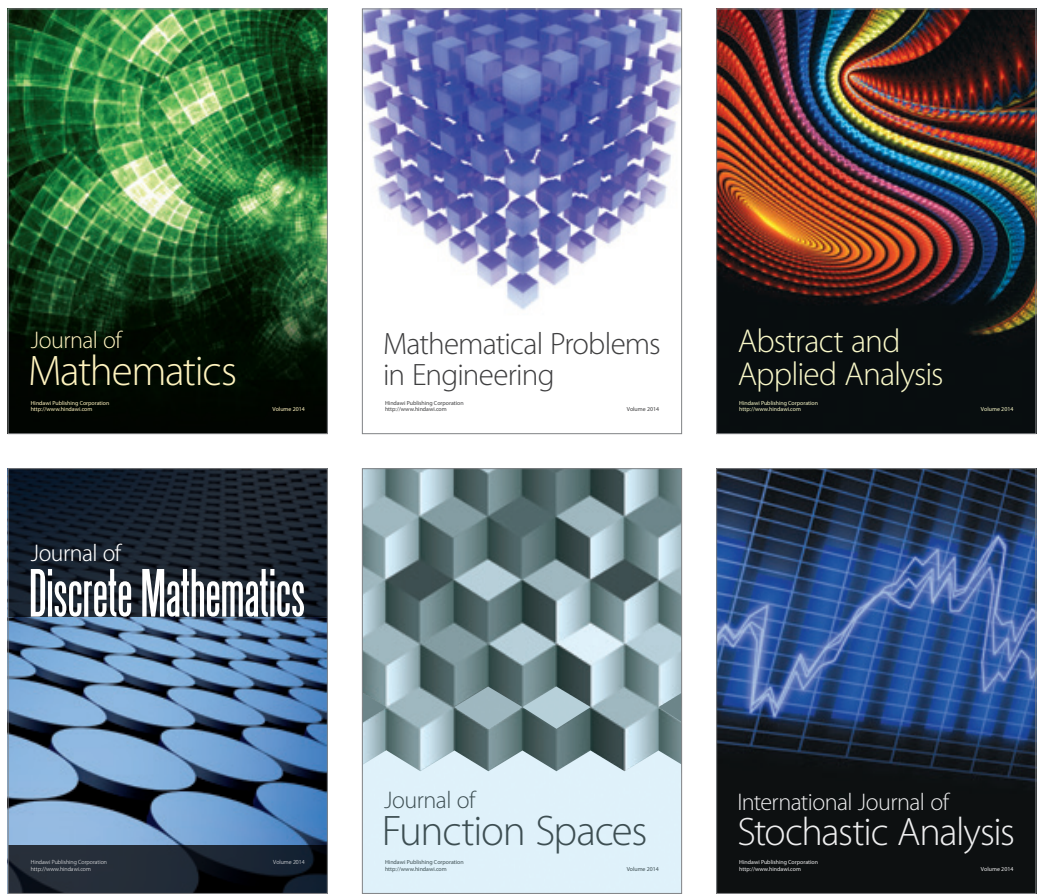

Journal of

Function Spaces

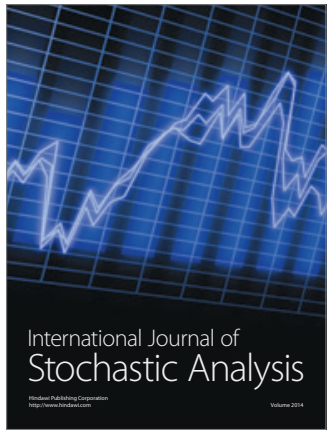

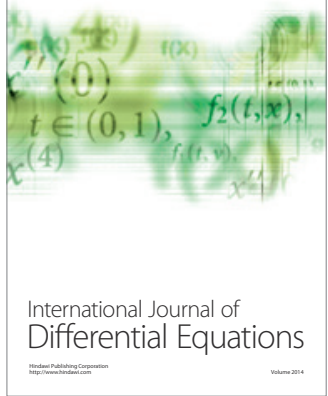
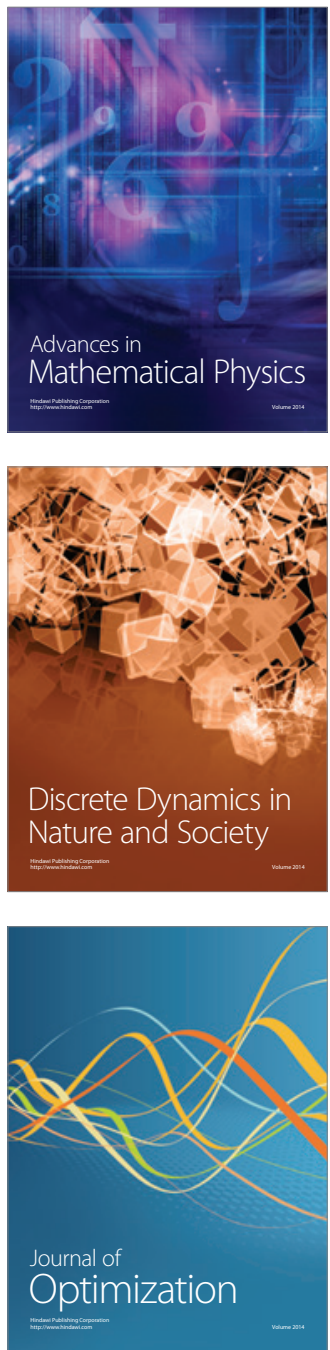